\title{
Altered renal FGF23-mediated activity involving MAPK and Wnt: effects of the Hyp mutation
}

\author{
Emily G Farrow, Lelia J Summers, Susan C Schiavi ${ }^{1}$, James A McCormick ${ }^{2}$, David H Ellison ${ }^{2}$ \\ and Kenneth E White
}

Department of Medical and Molecular Genetics, Indiana University School of Medicine, 975 West Walnut Street, IB130, Indianapolis, Indiana 46202, USA

${ }^{1}$ Genzyme, Corporation, Framingham, Massachusetts 01701-8861, USA

${ }^{2}$ Oregon Health and Science University, Portland, Oregon 97239, USA

(Correspondence should be addressed to K E White; Email: kenewhit@iupui.edu)

\begin{abstract}
Fibroblast growth factor-23 (FGF23), a hormone central to renal phosphate handling, is elevated in multiple hypophosphatemic disorders. Initial FGF23-dependent Erk1/2 activity in the kidney localizes to the distal convoluted tubule (DCT) with the co-receptor $\boldsymbol{\alpha}$-Klotho (KL), distinct from Npt2a in proximal tubules (PT). The Hyp mouse model of X-linked hypophosphatemic rickets $(\mathrm{XLH})$ is characterized by hypophosphatemia with increased Fgf23, and patients with XLH elevate FGF23 following combination therapy of phosphate and calcitriol. The molecular signaling underlying renal FGF23 activity, and whether these pathways are altered in hypophosphatemic disorders, is unknown. To examine Npt2a in vivo, mice were injected with FGF23. Initial p-Erk1/2 activity in the DCT occurred within $10 \mathrm{~min}$; however, Npt2a protein was latently reduced in the PT at 30-60 min, and was independent of Npt2a mRNA changes. $K L$-null mice had no DCT p-Erk1/2
\end{abstract}

staining following FGF23 delivery. Under basal conditions in Hyp mice, c-Fos and Egr1, markers of renal Fgf23 activity, were increased; however, KL mRNA was reduced $60 \%$ $(P<0 \cdot 05)$. Despite the prevailing hypophosphatemia and elevated Fgf23, FGF23 injections into Hyp mice activated p-Erk1/2 in the DCT. FGF23 injection also resulted in phospho- $\beta$-catenin ( $\mathrm{p}-\beta$-cat) co-localization with $\mathrm{KL}$ in wild-type mice, and Hyp mice demonstrated strong $\mathrm{p}-\beta$-cat staining under basal conditions, indicating potential crosstalk between mitogen-activated protein kinase and Wnt signaling. Collectively, these studies refine the mechanisms for FGF23 bioactivity, and demonstrate novel suppression of Wnt signaling in a KL-dependent DCT-PT axis, which is likely altered in XLH. Finally, the current treatment of phosphate and calcitriol for hypophosphatemic disorders may increase FGF23 activity.

Journal of Endocrinology (2010) 207, 67-75

\section{Introduction}

Fibroblast growth factor-23 (FGF23) has been identified as the key regulator of phosphate metabolism. The importance of FGF23 in phosphate metabolism is underscored by disorders of hypophosphatemia associated with increased circulating FGF23 including autosomal dominant hypophosphatemic rickets (ADHR), X-linked hypophosphatemic rickets (XLH), autosomal recessive hypophosphatemic rickets, tumor-induced osteomalacia, as well as through syndromes of reduced FGF23, primarily hyperphosphatemic tumoral calcinosis (TC). In situations of elevated FGF23, renal phosphate wasting occurs through down-regulation of the proximal tubule (PT) membrane type-IIa (Npt2a) and type-IIc (Npt2c) sodium phosphate co-transporters (Larsson et al. 2004, Shimada et al. 2004c). In parallel, FGF23 decreases the expression of PT vitamin D 1- $\alpha$-hydroxylase (CYP27b) and increases the expression of 24-hydroxylase (CYP24), thereby reducing the serum concentrations of $1,25(\mathrm{OH})_{2}$ vitamin D (Shimada et al. 2001). These changes in gene expression account for the paradoxical low-circulating $1,25(\mathrm{OH})_{2}$ vitamin $\mathrm{D}$ in the face of prevailing hypophosphatemia in syndromes associated with increased FGF23.

The co-receptor $\boldsymbol{\alpha}$-Klotho (KL) has been identified as necessary for FGF23 activity (Kuro-o 2006, Urakawa et al. 2006). The hypothesized mechanism for FGF23 bioactivity with $\mathrm{KL}$ is the recruitment of canonical FGF receptors (FGFRs) to form heteromeric complexes that then signal through the mitogen-activated protein kinase (MAPK) cascade and phospho-ERK1/2 (p-ERK1/2; Kuro-o 2006, Urakawa et al. 2006). In support of FGF23-KL interactions, the Fof23- and KL-null animals have highly similar biochemical and skeletal phenotypes (Shimada et al. 2004b, Sitara et al. 2004), and a recessive, inactivating mutation in the $K L$ gene resulted in impaired KL expression and a severe human TC phenotype, most likely due to end-organ resistance to FGF23 (Ichikawa et al. 2007). Although it has been shown that interactions between $\mathrm{KL}$ and a canonical 
FGFR are necessary to mediate FGF23 signaling in vitro, the mechanisms underlying FGF23 bioactivity in vivo are unclear, as $\mathrm{KL}$ localizes to the renal distal convoluted tubule (DCT) and FGF23 mediates its effects on NPT2a and vitamin D metabolizing enzymes within the PT (Shimada et al. 2001, Larsson et al. 2004). We previously demonstrated that initial renal FGF23-dependent activity, as measured by p-ERK1/2 expression, occurs in the DCT localizing with KL expression (Farrow et al. 2009). These findings indicate the presence of an intra-renal signaling axis controlling the biological effects of FGF23 on renal phosphate and vitamin D metabolism. Previous studies demonstrated a maximal decrease in serum phosphate concentrations at $8 \mathrm{~h}$ following FGF23 injection into mice (Shimada et al. 2004a). Additionally, acute administration of parathyroid hormone $(\mathrm{PTH})$ results in a decrease in Npt2a expression in the apical membrane $30 \mathrm{~min}$ after injection (Bacic et al. 2003). Although initial FGF23 activity occurs in the renal distal tubule within $10 \mathrm{~min}$ (Farrow et al. 2009), the temporal control of Npt2a and the downstream mediators of FGF23-dependent effects between the DCT and PT remain unclear.

$\mathrm{XLH}$ is the most common form of heritable hypophosphatemia (HYP-Consortium 1995), and most patients have chronically elevated serum FGF23 (Jonsson et al. 2003). The current therapy for XLH includes an oral regimen of phosphate and calcitriol; however, the treatment itself can have significant side effects including hyperparathyroidism (Lyles et al. 1982). Of significance, phosphate and 1,25(OH) vitamin D stimulate the production of Fgf23 in animals (Azam et al. 2003, Shimada et al. 2004c), and this therapeutic regimen has recently been shown to increase circulating FGF23 concentrations in XLH patients (Imel et al. 2010). The Hyp mouse model of XLH, in parallel with the human condition, has significantly elevated Fgf23 serum concentrations and Fgf23 bone mRNA expression (Liu et al. 2006b). These animals also increase FGF23 in response to dietary phosphate supplementation and vitamin D injections (Liu et al. 2006a). However, the molecular effects of chronically elevated FGF23 on the renal mechanisms of phosphate handling are unknown, and whether FGF23 is capable of further signaling effects in the face of elevated Fgf23 in Hyp is also unclear. Therefore, the goals of the present studies were to identify novel pathways involved in renal FGF23 bioactivity as a portion of a multinephron segment process, and to test whether FGF23dependent signaling is altered in syndromes associated with FGF23 excess.

\section{Materials and Methods}

\section{Animal studies}

All animal studies were performed according to the Indiana University Institutional Animal Care and Use Committee guidelines. Wild-type (WT) C57/Bl6, Hyp (Jackson Laboratories, Bar Harbor, ME, USA), or KL-null (Lexicon Genetics,
Inc., The Woodlands, TX, USA) mice were injected with either vehicle (saline) or $10 \mu \mathrm{g}$ recombinant FGF23 (gift from Genzyme Corp., Framingham, MA, USA) either i.v. or i.p. The $K L$-null mice carry the $\beta$-galactosidase gene cassette in place of mouse KL exons 2 and 3. Human FGF23 with the R176Q ADHR mutation was produced in insect cells (d-Mel-2), and is fully glycosylated. Animals were killed at 10, 30, and $60 \mathrm{~min}$ following injection. Kidneys were harvested for the preparation of RNA and immunofluorescence (IF) analyses, and serum was collected for serum FGF23 analysis.

\section{Cell culture}

HEK293 cells (American Type Culture Collection, Manassas, VA, USA) were cultured in DMEM/F-12 (Invitrogen) supplemented with 10\% fetal bovine serum (Hyclone, Hudson, NH, USA), $1 \mathrm{mM}$ sodium pyruvate, $2 \mathrm{mM}$ L-glutamine, and $25 \mathrm{mM}$ penicillin-streptomycin at $37^{\circ} \mathrm{C}$ and $5 \% \mathrm{CO}_{2}$. Cells stably expressing mKL were generated as described previously (Farrow et al. 2009). For bioactivity assays, mKL cells $\left(1.5 \times 10^{5}\right)$ were seeded in 12 -well plates. Cells were serum starved for a minimum of $2 \mathrm{~h}$ before treatment with vehicle or $100 \mathrm{ng} / \mathrm{ml} \mathrm{FGF23} \mathrm{for} \mathrm{0,} \mathrm{30,} \mathrm{or} 60 \mathrm{~min}$.

\section{Immunofluorescence}

Kidneys were fixed with $4 \%$ paraformaldehyde, mounted in OCT (Sakura Finetek, Torrance, CA, USA), and sliced into $10-\mu \mathrm{m}$ sections. Sections were treated with pepsin (Biocare Medical, Concord, CA, USA) for antigen retrieval, and were probed with anti-mouse KL (Santa Cruz Biotechnology, Santa Cruz, CA, USA), p-ERK1/2 (Cell Signaling, Danvers, MA, USA), phospho- $\beta$-catenin ( $\mathrm{p}-\boldsymbol{\beta}$-cat; Cell Signaling), or a highly specific antibody to mouse Npt2a (Custer et al. 1994) followed by incubation with the appropriate fluorescent secondary antibodies (Alexa Fluor, Invitrogen). Where indicated, immunofluorescent signal amplification was performed using the Tyramide signal amplification systems kit according to the manufacturer's directions (Perkin Elmer, Waltham, MA, USA). Slides were mounted using DAPI (Vector Laboratories, Burlingame, CA, USA). Imaging was performed using a Leica DM5000B fluorescent microscope (Leica Microsystems, Inc., Bannockburn, IL, USA), and SPOT camera and computer program (RTKE Diagnostic Instruments, Inc., Sterling Heights, MI, USA). A minimum of 3-4 animals per time point and 8-12 sections per animal were examined for each primary antibody set.

\section{Quantitative RT-PCR}

Kidneys were harvested for RNA using TRIzol (Invitrogen) according to the manufacturer's protocol. RNA was harvested from the cellular lysates using the RNeasy kit (Qiagen, Inc.). MAPK signal (PAMM-061) and signal transduction pathway finder (PAMM-014) array plates were obtained from SABiosciences (Frederick, MD, USA) and used according to 
the manufacturer's protocol. To confirm array results, RNA samples were tested with intron-spanning primers specific for mouse Egr1 mRNA (forward $5^{\prime}$-agccgagcgaacaaccctat- $3^{\prime}$, reverse $5^{\prime}$-cgccttctcattattcagagcg- $3^{\prime}$, probe $5^{\prime}$-agcacctgaccacagagtccttttctgaca- $3^{\prime}$ ), mouse c-Fos (Applied Biosystems, Carlsbad, CA, USA), and mouse KL (forward $5^{\prime}$-cagctccaggctcgggta- $3^{\prime}$, reverse $5^{\prime}$-aggtgttgtagagatgccagacttt- $3^{\prime}$, probe $5^{\prime}$-ttgcccacaacctacttttggctcatg- $3^{\prime}$ ), and mouse actin (forward $5^{\prime}$-ggctcctagcaccatgaag- $3^{\prime}$, reverse $5^{\prime}$-cgccttctcattattcagagcg- $3^{\prime}$, probe $5^{\prime}$-agcacctgaccacagagtcctttctgaca- $3^{\prime}$ ) was used as an internal control. Intron-spanning primers specific for human EGR1 mRNA (forward $5^{\prime}$-ggacacgggcgagcag- $3^{\prime}$, reverse $5^{\prime}$-cgttgttcagagagatgtcagga- $3^{\prime}$, probe $5^{\prime}$-cctacgagcacctgaccgcagagtct- $\left.3^{\prime}\right)$, human c-FOS (Applied Biosystems), and human actin (forward $5^{\prime}$-ggcacccagcacaatgaag- $3^{\prime}$, reverse $5^{\prime}$-gccgatccacacggagtact- $3^{\prime}$, probe $5^{\prime}$-tcaagatcattgctcctcctgagcgc- $3^{\prime}$ ) were used for in vitro experiments with HEK293 cells. The TaqMan One-Step RT-PCR kit (Applied Biosystems) was used to perform quantitative RT-PCR (qPCR). PCR conditions for all experiments were $30 \mathrm{~min}$ $48^{\circ} \mathrm{C}, 10 \mathrm{~min} 95^{\circ} \mathrm{C}$, followed by 40 cycles of $15 \mathrm{~s} 95^{\circ} \mathrm{C}$ and $1 \mathrm{~min} 60^{\circ} \mathrm{C}$. The data were collected and analyzed by the 7500 Real-Time PCR system and software (Applied Biosystems). The expression levels of Egr1, c-Fos, Klotho, and $\beta$-actin mRNAs were calculated relative to WT littermates or vehicle-treated controls. All primer sets were tested for specific amplification of mRNA by parallel analyses of controls that included omitting RT or template, and resulted in no fluorescent signal detection. Each RNA sample was analyzed in at least triplicate, and each experiment was performed independently at least three times. The $2^{-\Delta \Delta C_{\mathrm{T}}}$ method described by Livak was used to analyze the data (Livak \& Schmittgen 2001).

\section{FGF23 serum measurements}

Serum intact FGF23 concentrations were assessed using an ELISA according to the manufacturer's protocol (Kainos Laboratories Int'l, Tokyo, Japan). This two-site, monoclonal antibody ELISA has previously been shown to recognize rodent Fgf23 (Yamazaki et al. 2002).

\section{Statistical analysis}

Statistical analysis of the data was performed by Student's $t$-test, and significance for all the tests was set at $P<0 \cdot 05$. Data are presented as means \pm s.E.M.

\section{Results}

\section{Temporal control of Npt2a by FGF23}

We previously demonstrated that initial FGF23 bioactivity, as assessed by ERK1/2 phosphorylation (p-ERK1/2), co-localizes with KL in the renal DCT within $10 \mathrm{~min}$ after injection (Farrow et al. 2009). However, it has been shown that FGF23 delivery results in a latent reduction in serum phosphate concentrations, with a maximum decrease apparent at $8 \mathrm{~h}$ (Shimada et al. 2004a). Thus, the temporal control of Npt2a in the renal PT by FGF23 is not clear. To determine the effect of FGF23 on Npt2a expression, WT mice were injected with vehicle or $10 \mu \mathrm{g}$ recombinant human FGF23 for 10, 30, and $60 \mathrm{~min}$, and the kidneys were harvested for RNA and for IF analyses. Npt2a expression was diminished in the PT apical membrane within $30 \mathrm{~min}$ following FGF23 injection, and remained decreased $60 \mathrm{~min}$ after injection (Fig. 1a). No change in Npt2a protein expression was observed in vehicleinjected animals at any time point (Fig. 1a). Kidney sections were co-stained with actin to delineate the apical membrane, which confirmed that the majority of $\mathrm{Npt} 2 \mathrm{a}$ was removed from the apical membrane by $60 \mathrm{~min}$ (Fig. 1a). Robust p-ERK1/2 staining was confirmed to localize to the renal DCT at $10 \mathrm{~min}$ (Fig. 1b). Immunofluorescent signal amplification was used to test for p-ERK1/2 expression in the PT at 30 and $60 \mathrm{~min}$. However, we were unable to identify a p-ERK1/2 signal co-localized with $\mathrm{Npt} 2 \mathrm{a}$ at these time points (Fig. 1b).

The change in Npt2a protein localization within the apical membrane was independent of a transcriptional change, as a reduction in Npt2a mRNA was not detected between vehicle- and FGF23-injected animals $(P=0 \cdot 7$; Fig. 1c). In contrast, the positive control for FGF23 activity, early growth response gene-1 (Egr1) mRNA was dramatically increased ( 50-fold; $P<0.05$; not shown). Thus, initial FGF23 activity in the DCT through p-ERK1/2 occurs within 10 min, whereas Npt2a protein expression in the PT is a latent effect (30-60 min) that does not require changes in Npt2a mRNA levels.

To test whether KL was required for initial FGF23 activity through MAPK in the DCT, $K L$-null mice were injected with $10 \mu \mathrm{g}$ FGF23 and killed at $10 \mathrm{~min}$. These analyses indicated that ERK1/2 was not activated in the tubule segments of vehicle-injected WT mice (Fig. 1d, left), or in the $K L$-null mice that express a surrogate marker for KL expression, $\beta$-galactosidase (Fig. 1d, right). The positive control WT mice showed strong p-ERK1/2 activity (Fig. 1d, middle).

\section{FGF23-mediated signaling under normal conditions and in disease}

To identify genes responsive to FGF23, we tested for changes in transcription factors other than Egr1 that are known to be downstream of canonical FGF signaling, including c-Fos (Kang et al. 2005). In this regard, WT mice were injected with either vehicle or $10 \mu \mathrm{g}$ recombinant human FGF23 i.p. ( $n=3-4$ /group) and killed at $60 \mathrm{~min}$. qPCR was performed for c-Fos and Egr1 on RNA isolated from the kidneys of injected animals. c-Fos showed a 13-fold increase in mRNA expression. The positive control, Egr1, increased over 70-fold in FGF23-injected mice when compared with the vehicletreated controls $(P<0 \cdot 02 ;$ Fig. 2 a). To confirm these results, HEK293 cells stably expressing $\mathrm{mKL}$ were treated with 

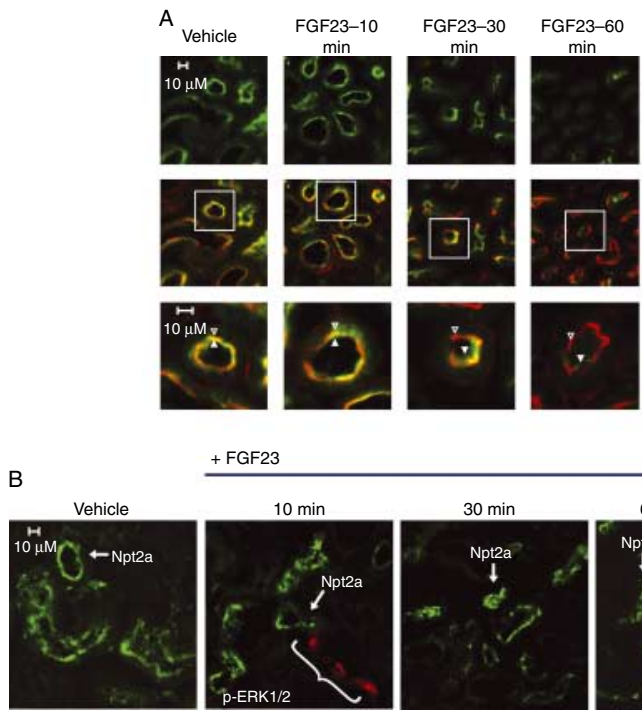

+ FGF23
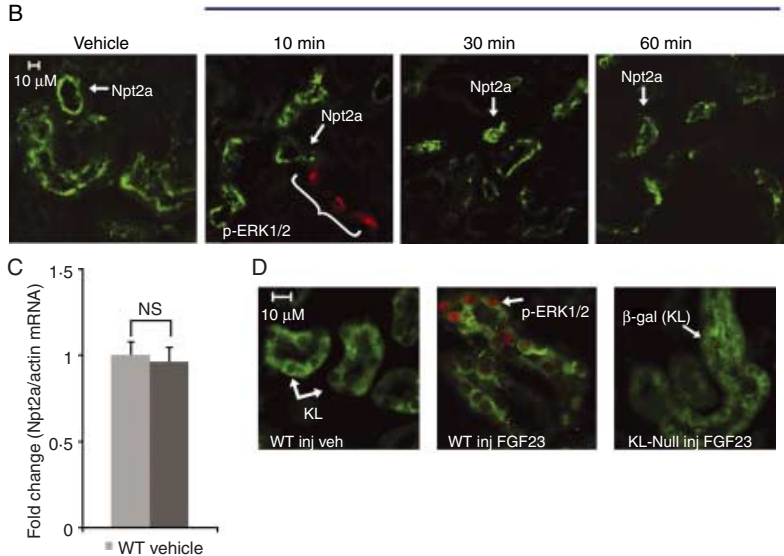

D
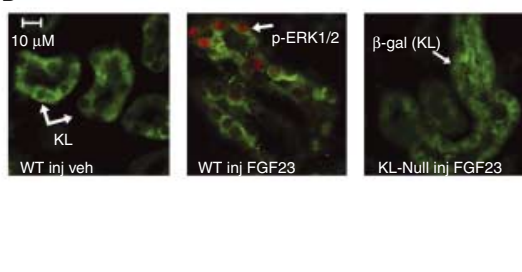

Figure 1 Control of Npt2a by FGF23. (a) Mice were injected with FGF23 or vehicle for 10,30 , or 60 min. Under standard staining conditions, Npt2a expression was tested. Npt2a was reduced 30-60 min following injection with FGF23 (top row). Sections were co-stained with actin (red), to mark the apical membrane, and co-localization of Npt2a in the apical membrane was detected (yellow). By $60 \mathrm{~min}, \mathrm{Npt} 2 \mathrm{a}$ was not detected in the apical membrane (middle row). Higher magnification of the proximal tubule shows the progressive removal of Npt2a from the apical membrane (bottom row). (b) Sections were co-stained for Npt2a and $p$-ERK1/2. p-ERK1/2 activity was absent in the vehicle-injected animals. At the 10 -min time point, p-ERK1/2 was confirmed in the animals, and localization was distinct from Npt2a expression in the PT; no p-ERK $1 / 2$ activity was detected in the PT at any time point tested. At the 30- and 60-min time points, Npt2a staining was not detected as reduced (as in 1a) due to the effects of signal amplification for Npt2a and p-ERK1/2. (c) The reduction in Npt2a at 60 min was independent of transcription, as no change in Npt2a mRNA was detected $(P=0 \cdot 7)$. (d) WT or $K L$-null mice were injected with vehicle or $10 \mu \mathrm{g}$ FGF23 (WT vehicle, left panel; WT FGF23, middle panel) to test for renal MAPK activity. In the $K L$-null animals, the $L a c Z$ gene is inserted into the Klotho locus, which enabled $\beta$-galactosidase ( $\beta$-gal) immunofluorescence to be used as a surrogate marker for KL (green, right panel). Following FGF23 delivery to $K L$-null mice, p-ERK $1 / 2$ activity was absent in the DCT labeled with $\beta$-gal (KL) (right panel). As confirmed, strong p-ERK1/2 activity co-localized with KL in WT animals injected with FGF23 (middle). Full colour version of this figure available via http://dx.doi.org/10.1677/JOE-10-0181.

$100 \mathrm{ng} / \mathrm{ml}$ recombinant human FGF23 for 0, 30, and $60 \mathrm{~min}$. A time-dependent increase was observed for both $c$-Fos and EGR 1 mRNAs with a maximum increase of 30- and 200-fold respectively $(P<0 \cdot 05$; Fig. $2 \mathrm{~b})$. c-Fos is known to be downstream of ERK1/2 in canonical FGF signaling; however, it has not been reported previously as responsive to FGF23. Taken together, these in vivo and in vitro results confirm the utilization of c-Fos as a marker of FGF23 bioactivity.

Patients with XLH have increased circulating FGF23; however, the molecular consequences in renal phosphate handling due to this elevation are unclear. Building upon the results directly above, we next tested basal levels of genes downstream of FGF23 activity in the Hyp model of XLH. These animals are characterized by hypophosphatemia and significantly increased serum Fgf23 levels ( $\sim 12$-fold over WT littermates $P<0 \cdot 05$; Fig. 3a); however, whether this leads to alterations in markers of FGF23 activity are unknown. Using c-Fos and Egr1 to test FGF-dependent activity in kidneys from $H y p$ mice, we observed a $200 \%$ increase in the expression of these genes in Hyp mice in the basal state compared with sex- and age-matched WT littermates $(P<0 \cdot 05$; Fig. 3b), suggesting that FGF23-dependent activity is enhanced in $H y p$ mice. Furthermore, $K L$ mRNA was reduced by $60 \%$ in these mice compared with WT littermates $(P<0 \cdot 05$; Fig. $3 \mathrm{~b})$, indicating that a potential compensatory mechanism is induced in an attempt to down-regulate FGF23-dependent signaling.

\section{MAPK activity in the Hyp mouse model}

The standard treatment for patients with XLH is a combination therapy of phosphate and calcitriol, which is used to raise serum phosphate. This regimen has recently been associated with increasing circulating FGF23
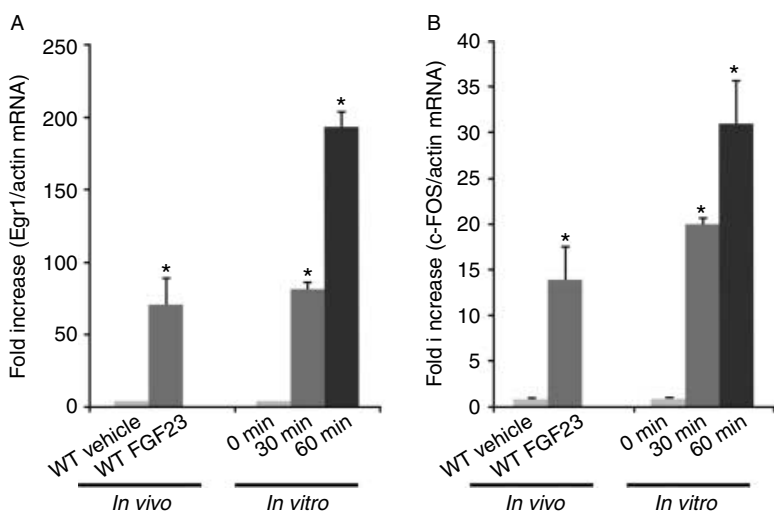

Figure 2 In vivo and in vitro MAPK signaling. (a) To test for novel markers of renal activity, mice were injected with $10 \mu \mathrm{g}$ FGF23 i.p. for $1 \mathrm{~h}$, and the kidneys were harvested for RNA preparation and qPCR. Egr1, measured as a positive control, was increased 70-fold over vehicle-injected mice. c-Fos was increased 13-fold $\left({ }^{*} P<0 \cdot 05\right)$. (b) HEK293 cells stably expressing $\mathrm{mKL}$ were treated with $100 \mathrm{ng} / \mathrm{ml} \mathrm{FGF23} \mathrm{for} 30$ and $60 \mathrm{~min}$. EGR1 mRNA increased 80 - and 200 -fold at 30 and 60 min respectively in the FGF23-treated mKL cells compared with vehicle-treated control $\left({ }^{*} P<0 \cdot 05\right)$. C-Fos increased $20-$ and $30-$ fold at 30 and $60 \mathrm{~min}$ respectively $(P<0 \cdot 05)$. 

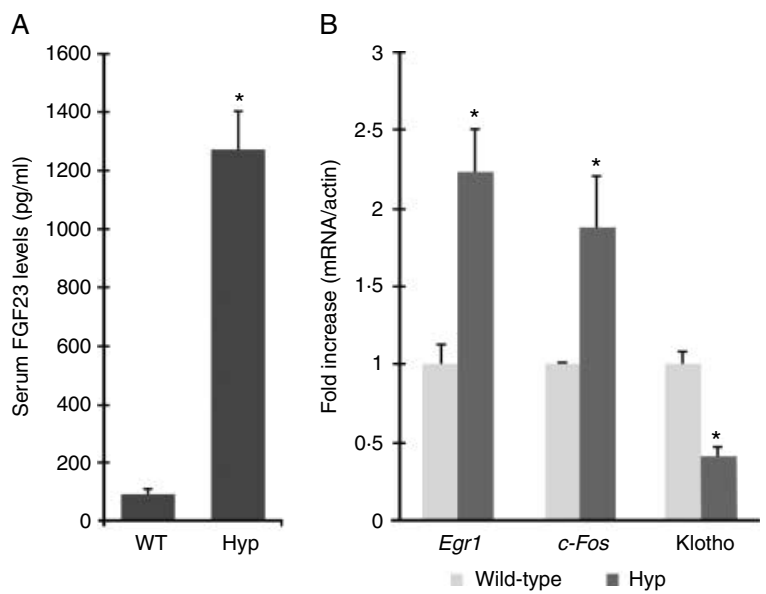

Figure 3 MAPK signaling in Hyp mice. (a) Hyp mice had intact serum FGF23 levels $\sim 12$-fold higher than WT controls $\left({ }^{*} P<0 \cdot 05\right)$; (b) Hyp mice had a 200\% increase in Egr1 and c-Fos mRNAs, and Klotho expression was reduced by $60 \%$ compared with wild-type controls $\left({ }^{*} P<0 \cdot 05\right)$.

concentrations in patients with XLH (Imel et al. 2010); however, the molecular effects of elevated FGF23 on the renal mechanisms of phosphate handling in this disorder are unknown. To test whether Hyp mice are able to respond to increased levels of FGF23, Hyp mice were injected with vehicle or FGF23 (10 $\mu \mathrm{g} / \mathrm{animal})$. Despite the decreased mRNA levels of KL, KL was detectable by IF (Fig. 4); however, no p-ERK1/2 activity was detected in the basal state in Hyp mice. In contrast, Hyp mice injected with FGF23 demonstrated strong p-ERK1/2 staining (Fig. 4), and this activity co-localized with KL in the renal DCT. These findings demonstrate that $H y p$ mice are capable of responding to increased serum FGF23 levels, consistent with the idea that the current treatment for XLH could be associated with elevated renal FGF23 bioactivity.

\section{FGF23-dependent effects on renal Wnt signaling}

To screen for novel pathways involved in renal FGF23dependent signaling, PCR-based array technology was utilized in initial experiments. This Pathway-Finder array contains primers recognizing 84 genes from 18 signaling pathways, including the positive control for Fgf23 activity, Egr1. WT mice were injected with $10 \mu \mathrm{g}$ recombinant human FGF23 and killed at $60 \mathrm{~min}$, and the kidneys were harvested for RNA preparation. Consistent with our earlier results, Egr1 mRNA was elevated $\sim 20$-fold in the array following injection. Unexpectedly, these initial analyses also identified Engrailed-1 (En1) and Wnt1 as elevated following injection with 3.5- and 5-fold increases in mRNA of FGF23injected mice compared with vehicle-injected mice (not shown). En1 is a well-characterized repressor of Wnt signaling (Bachar-Dahan et al. 2006). The role of Wnts as well as the localization of this signaling cascade in FGF23-dependent renal activity has not been previously defined.

To build upon the mRNA results at the protein level, FGF23-dependent suppression of renal Wnt signaling was tested. In this regard, kidney sections from vehicle- and FGF23-injected mice $(10 \mu \mathrm{g} / \mathrm{animal}$ for $10 \mathrm{~min})$ were co-stained for $p$ - $\beta$-cat, a well-characterized marker of suppression of Wnt signaling, as well as for KL. A strong $\mathrm{p}-\beta$-cat signal was only detected in the renal glomeruli of the vehicle-injected animals (Fig. 5a). In contrast, p- $\beta$-cat signaling was observed in the glomeruli and also co-localized with KL in the renal DCT in the FGF23-injected animals (Fig. $5 b$ ). Thus, in a similar manner to p-ERK1/2, p- $\beta$-cat activity was spatially separate from PT Npt2a expression.

Owing to the strong Wnt signaling response in WT mice, we next tested for the presence of $\mathrm{p}-\beta$-cat signaling in $H_{y p}$ mice, which would be consistent with the increased Egr 1 and c-Fos mRNAs indicating elevated MAPK signaling in the basal state (see Fig. 3b). Indeed, uninjected Hyp mice expressed robust $\mathrm{p}-\beta$-cat in the DCT co-localized with KL (Fig. 6). Furthermore, $\mathrm{p}-\beta$-cat was specifically co-localized with the sodium chloride co-transporter (NCC), a reliable DCT marker (Bostanjoglo et al. 1998). Importantly, the DCT-specific staining pattern for $\mathrm{p}-\beta$-cat paralleled that of WT mice injected with FGF23, i.e. being distinct from Npt2a in the PT (Fig. 6). Thus, these findings revealed that FGF23 acts to suppress Wnt signaling in the renal DCT, further supporting an intra-renal axis for FGF23 activity.
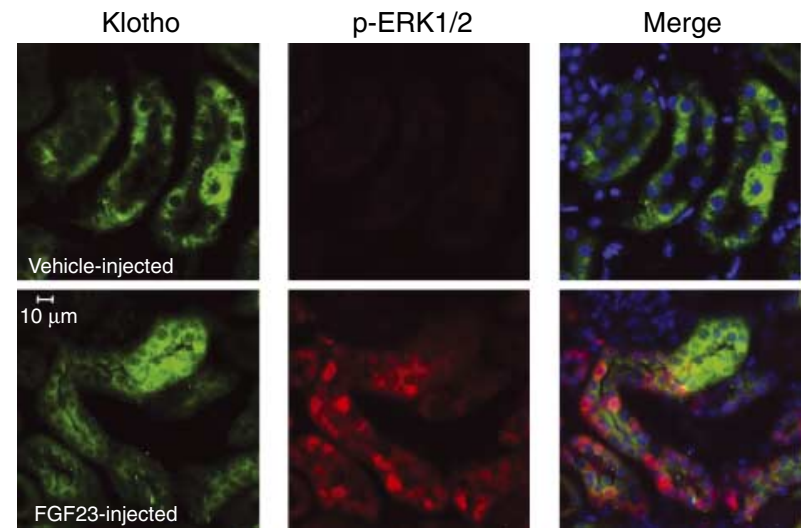

Figure 4 Response of Hyp to FGF23 delivery. Results obtained from vehicle-injected Hyp mice are shown in the upper panels, and from FGF23-injected Hyp mice shown in the lower panels at $10 \mathrm{~min}$ post injection. Phospho-ERK1/2 staining was only observed in the FGF23-injected animals; Klotho (KL) was positive in both vehicleand FGF23-injected animals. p-ERK1/2 staining (red) localized to the nucleus in the same nephron segment as KL (green) in FGF23injected mice as shown by p-ERK1/2 and KL co-staining ('Merge' column; arrows show positive nuclear $\mathrm{p}$-ERK1/2 co-localized with $\mathrm{KL}$ ). Nuclei were stained blue using DAPI in the Merge column. Full colour version of this figure available via http://dx.doi.org/10.1677/ JOE-10-0181. 
A
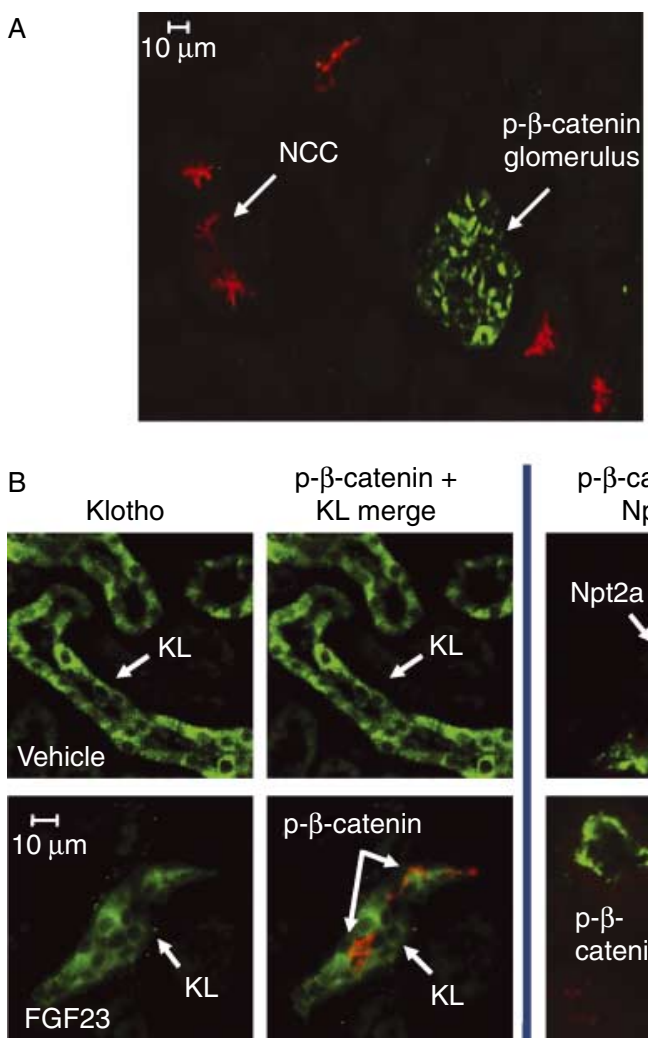

\begin{abstract}
$\mathrm{p}-\beta$-catenin + $\mathrm{KL}$ merge
\end{abstract}
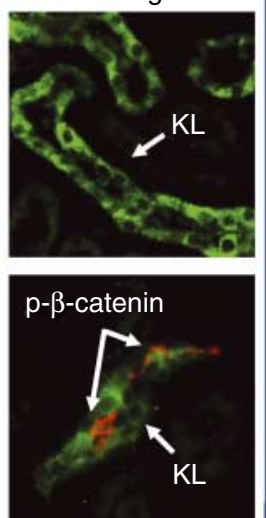

$\mathrm{p}-\beta$-catenin + Npt2a
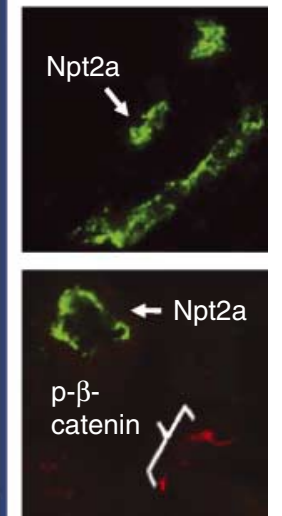

Figure 5 Wnt pathway in renal FGF23 signaling. (a) Strong $p$ - $\beta$-catenin signaling was detected in the glomeruli of all animals (green), separate from the DCT, which was demarcated using antiNCC IF (red). (b) $p$ - $\beta$-catenin signaling was tested by IF in vehicleand FGF23-injected mice at 10 min following injection. $\mathrm{p}$ - $\beta$-catenin signaling was not detected in the vehicle-injected animals. In the FGF23-injected animals, p- $\beta$-catenin (red) was detected in the distal tubule, co-localized with Klotho (green). This activity did not co-localize with Npt2a in the renal PT (right column). Full colour version of this figure available via http://dx.doi. org/10.1677/JOE-10-0181.

\section{Discussion}

FGF23 controls renal phosphate handling by directing the membrane localization of $\mathrm{Npt} 2 \mathrm{a}$ and $\mathrm{Npt} 2 \mathrm{c}$ (Larsson et al. 2004, Shimada et al. 2004c) in the PT. Previously, we demonstrated that initial FGF23-dependent MAPK activity occurs within minutes in the renal DCT (Farrow et al. 2009). It has also been shown that serum phosphate levels are maximally decreased in mice $\sim 9 \mathrm{~h}$ after FGF23 injection (Shimada et al. 2004a). Herein, we show that following FGF23 delivery, in a manner similar to PTH (Bacic et al. 2003), Npt2a protein expression is decreased in the PT by $30 \mathrm{~min}$ and fully at $60 \mathrm{~min}$ (Fig. 1a), and is associated with p-ERK1/2 activity in the DCT (Fig. 1b). This relatively rapid reduction in Npt2a is likely due to the endocytic retrieval of the co-transporter from the apical membrane, as no transcriptional changes in $\mathrm{Npt} 2 \mathrm{a}$ were detected at $60 \mathrm{~min}$ (Fig. 1c). Although FGF23 rapidly down-regulates $\mathrm{Npt} 2 \mathrm{a}$, changes in serum phosphate concentrations and $1,25(\mathrm{OH})_{2}$ vitamin $\mathrm{D}$ are known to be latent (Shimada et al. 2004a). Our findings support the idea that elevations in FGF23 may also be associated with indirect endocrine changes that manifest over longer time intervals, as opposed to balancing minute to minute serum phosphate changes. Importantly, FGF23 activity through MAPK in the DCT was dependent upon the presence of KL. In this regard, p-ERK1/2 activity was not detected that co-localized with DCT segments expressing a surrogate marker for KL, $\beta$-galactosidase (Fig. 1d) in $K L$-null animals injected with FGF23. These results are consistent with the fact that KL and a canonical FGFR are required for FGF23-dependent MAPK signaling (Urakawa et al. 2006). Furthermore, the lack of FGF23-dependent p-ERK1/2 activity in the DCT in the absence of KL provides mechanistic support for a report demonstrating a likely end-organ deficiency in FGF23dependent signaling in a patient with severe TC harboring a homozygous $K L$ loss-of-function missense mutation (His193Arg; Ichikawa et al. 2007).

We have now demonstrated the presence of early FGF23 signaling in the DCT with both p-ERK1/2 and Wnt involvement (see below). Nonetheless, the mechanisms underlying a potential transfer of FGF23 bioactivity across a spatial separation to the PT remain unclear. Although the second step or process following initial FGF23 signaling is unknown, our results support the fact that it likely occurs relatively quickly (under $30 \mathrm{~min}$ ) after initial signaling (5 min), although physiological changes in serum phosphate are known to occur over at least 8-9 h (Shimada et al. 2004a). Collectively, these findings provide a narrowed time frame for the identification of candidate pathways guiding intra-renal FGF23-dependent activity. At this time, however, the possibility that FGF23 has a direct effect in the PT that does not involve p-ERK1/2 (or Wnts) cannot be ruled out.

To test for additional markers of FGF23 bioactivity, the transcription factor c-Fos was identified as being upregulated following FGF23 delivery in vivo and in vitro (Fig. 2b). Although c-Fos is known to be downstream of the MAPK
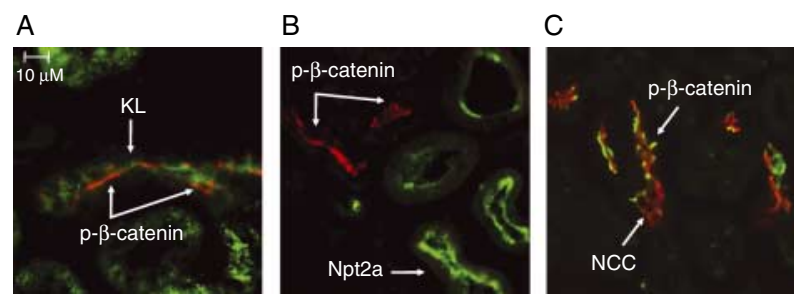

Figure 6 Renal $p$ - $\beta$-catenin expression in Hyp mice. (a) p- $\beta$-catenin (red) was readily detectable in kidneys from Hyp mice in the basal state, localized to the renal DCT with KL (green staining; left panel); (b) p- $\beta$-catenin (red) was spatially separate from Npt2a (green; middle panel). (c) In the Hyp basal state, strong $\mathrm{p}$ - $\beta$-catenin signal (green) was co-localized with NCC, a marker for the renal DCT (red; right panel). Full colour version of this figure available via http://dx. doi.org/10.1677/JOE-10-0181 
pathway initiated by canonical FGF signaling (autocrine and paracrine FGFs), it had not previously been associated with renal FGF23 bioactivity. Hyp mice have significantly elevated serum FGF23 and hypophosphatemia, and are a well-established model of the human syndrome XLH. The standard treatment for XLH currently includes supplemental oral phosphate and high-dose calcitriol (Tenenhouse \& Econs 2001). This therapeutic regimen has now been realized to result in further increases in circulating FGF23 in this patient group (Imel et al. 2010). Similarly, administration of phosphate or vitamin D potently increases FGF23 production in WT mice and in Hyp mice (Azam et al. 2003, Shimada et al. 2004c). Although the Hyp mouse has been well studied, the levels of relative renal FGF23 bioactivity under a state of chronic hypophosphatemia in this model remained unclear. We therefore examined FGF23 signaling in Hyp mice in the basal state and found that the markers of MAPK signaling, Egr1 and c-Fos mRNAs, were elevated in kidneys from Hyp mice compared with WT littermates (Fig. 3b). These findings are consistent with the idea that FGF23-dependent signaling is increased in Hyp mice under 'normal' circumstances, consistent with their significantly increased circulating Fgf23 (Fig. 3a; Liu et al. 2006b). Although renal $K L$ mRNA concentrations were reduced by 60\% in Hyp mice compared with WT littermates, when $H_{y p}$ mice were injected with FGF23, robust p-ERK1/2 signal was detected in the DCT with KL, similar to the response observed in WT mice (Fig. 1d). Consistent with our findings in Hyp mice, KL mRNA was reported to be the most significant downregulated kidney gene in a markedly hypophosphatemic transgenic mouse over-expressing human FGF23 in osteoblasts and osteocytes (Marsell et al. 2008). Conversely, KL transcripts were upregulated in a model of hyperphosphatemic TC, the GALNT3-null mouse (Ichikawa et al. 2009), which suggests that FGF23 may potentially be regulated at the level of its receptor complex in kidney depending upon phosphate status. Taken together, these results indicate that in the basal state, $H y p$ mice have altered renal MAPK activity, but overall, FGF23-dependent signaling remains intact. These findings have implications for $\mathrm{XLH}$, as increased FGF23 following treatment could suppress renal $1,25(\mathrm{OH})_{2}$ vitamin $\mathrm{D}$ production by decreasing 25 -vitamin $\mathrm{D}$ $1-\alpha$-hydroxylase and increasing 24 -hydroxylase expression, or have direct actions on the parathyroid, further leading to a scenario for secondary hyperparathyroidism, a common and often severe complication of XLH (Tenenhouse \& Econs 2001).

To screen for novel pathways associated with FGF23 renal activity, we used an array approach and identified markers of suppressed Wnt signaling. Wnt signaling is critical for proper kidney development (Herzlinger et al. 1994, Stark et al. 1994), and altered Wnt activity has been implicated in the development of several renal diseases including renal cell carcinoma and cystic kidney disease (Kim et al. 1999, 2000). Importantly, following FGF23 delivery, $\mathrm{p}-\beta$-cat signaling co-localized to the DCT with KL and with NCC (Figs $5 b$ and 6) within the same time course for DCT p-ERK1/2 expression (10 min). In Hyp mice in the basal state, p-ERK1/2 was not detected by IF (Fig. 4), consistent with the modest increases in Egr1 and $c$-Fos mRNAs (Fig. 3b). However, in the basal state, strong p- $\beta$-cat signaling was detected in the DCT of Hyp mouse, indicating that $\mathrm{p}-\beta$-cat may be a sensitive marker in XLH and in disease models of altered FGF23 activity.

The localization of Wnt signaling has not been defined in terms of renal phosphate homeostasis in vivo, and its role in the DCT for normal phosphate handling is unclear. It was reported that GSK3- $\beta$ phosphorylation was increased in vitro in cells treated with FGF23 and a recombinant KL (Medici et al. 2008) in parallel with p-ERK1/2 (Medici et al. 2008, Farrow et al. 2009). In canonical Wnt signaling, GSK3- $\beta$ is upstream of $p-\beta$-cat, consistent with our results that $p-\beta$-cat is localized to the DCT with p-ERK following FGF23 delivery, spatially distinct from Npt2a in the PT. Complementary signaling between the FGF-dependent MAPK and Wnt pathways has been well documented during development (ten Berge et al. 2008); however, the molecules at the origin of the branching point between these two pathways is less clear. Recent reports indicated that GSK3- $\beta$ may serve as a mediator between FGF and non-canonical Wnt signaling during carcinogenesis (Katoh 2006), and during osteoblast proliferation AKT may signal between MAPK and Wnts (Raucci et al. 2008). Thus, our identification of FGF23dependent control of Wnt activity in the kidney may also be useful for the further understanding of MAPK-Wnt interactions. Genes known to be involved in Wnt and FGF signaling are also altered in Hyp mouse bone (Liu et al. 2009); thus in addition to renal pathogenesis, these systems may also be associated with increased skeletal Fgf23 (Liu et al. 2003).

In summary, we have identified novel markers of renal FGF23 bioactivity, have defined the temporal control of Npt2a by FGF23, and have localized alterations in MAPK and Wnt signaling to the renal DCT in the Hyp mouse. These studies have important implications for understanding the altered FGF23-dependent signaling in XLH, as well as in other disorders associated with elevated FGF23.

\section{Declaration of interest}

E G F, L J S, J A M, and D H E have nothing to declare. S C S is employed by Genzyme, Corp. K E W receives research funding from Genzyme and royalties for licensing the FGF23 gene to Kyowa Hakko Kirin, Ltd.

\section{Funding}

The authors would like to acknowledge support from NIH grants DK051496 (D H E) and DK063934 and an ARRA Supplement to DK063934 (K E W), the Showalter Foundation, Genzyme Corporation, the Indiana Genomics Initiative (INGEN) of Indiana University supported in part by the Lilly Endowment, Inc. E G F is supported in part by a National Kidney Foundation (NKF) Research Fellowship. 


\section{Acknowledgements}

We would like to thank Drs Heini Murer and Jürg Biber at the Institute of Physiology and Zurich Center for Integrative Human Physiology, Zurich, Switzerland, for generously providing the anti-Npt2a antibody.

\section{References}

Azam N, Zhang MY, Wang X, Tenenhouse HS \& Portale AA 2003 Disordered regulation of renal 25-hydroxyvitamin D-1alpha-hydroxylase gene expression by phosphorus in X-linked hypophosphatemic (hyp) mice. Endocrinology 144 3463-3468. (doi:10.1210/en.2003-0255)

Bachar-Dahan L, Goltzmann J, Yaniv A \& Gazit A 2006 Engrailed-1 negatively regulates beta-catenin transcriptional activity by destabilizing beta-catenin via a glycogen synthase kinase-3beta-independent pathway. Molecular Biology of the Cell 17 2572-2580. (doi:10.1091/mbc.E06-01-0052)

Bacic D, Schulz N, Biber J, Kaissling B, Murer H \& Wagner CA 2003 Involvement of the MAPK-kinase pathway in the PTH-mediated regulation of the proximal tubule type $\mathrm{IIa} \mathrm{Na}^{+} / \mathrm{Pi}$ cotransporter in mouse kidney. Pflügers Archiv 446 52-60. (doi:10.1007/s00424-002-0969-8)

Bostanjoglo M, Reeves WB, Reilly RF, Velazquez H, Robertson N, Litwack G, Morsing P, Dorup J, Bachmann S \& Ellison DH 1998 11Beta-hydroxysteroid dehydrogenase, mineralocorticoid receptor, and thiazide-sensitive $\mathrm{Na}-\mathrm{Cl}$ cotransporter expression by distal tubules. Journal of the American Society of Nephrology 9 1347-1358.

Custer M, Lotscher M, Biber J, Murer H \& Kaissling B 1994 Expression of $\mathrm{Na}-\mathrm{P}$ (i) cotransport in rat kidney: localization by RT-PCR and immunohistochemistry. American Journal of Physiology 266 F767-F774.

Farrow EG, Davis SI, Summers LJ \& White KE 2009 Initial FGF23-mediated signaling occurs in the distal convoluted tubule. Journal of the American Society of Nephrology 20 955-960. (doi:10.1681/ASN.2008070783)

Herzlinger D, Qiao J, Cohen D, Ramakrishna N \& Brown AM 1994 Induction of kidney epithelial morphogenesis by cells expressing Wnt-1. Developmental Biology 166 815-818. (doi:10.1006/dbio.1994.1360)

HYP-Consortium 1995 A gene (PEX) with homologies to endopeptidases is mutated in patients with X-linked hypophosphatemic rickets. The HYP Consortium. Nature Genetics 11 130-136. (doi:10.1038/ng1095-130)

Ichikawa S, Imel EA, Kreiter ML, Yu X, Mackenzie DS, Sorenson AH, Goetz R, Mohammadi M, White KE \& Econs MJ 2007 A homozygous missense mutation in human KLOTHO causes severe tumoral calcinosis. Journal of Clinical Investigation 117 2684-2691. (doi:10.1172/JCI31330)

Ichikawa S, Sorenson AH, Austin AM, Mackenzie DS, Fritz TA, Moh A, Hui SL \& Econs MJ 2009 Ablation of the Galnt3 gene leads to lowcirculating intact fibroblast growth factor 23 (Fgf23) concentrations and hyperphosphatemia despite increased Fgf23 expression. Endocrinology 150 2543-2550. (doi:10.1210/en.2008-0877)

Imel EA DL, Hui SL, Carpenter TO \& Econs MJ 2010 Treatment of XLH with calcitriol and phosphate increases circulating FGF23 concentrations. Journal of Clinical Endocrinology and Metabolism 95 1846-1850. (doi:10.1210/ jc.2009-1671)

Jonsson KB, Zahradnik R, Larsson T, White KE, Sugimoto T, Imanishi Y, Yamamoto T, Hampson G, Koshiyama H, Ljunggren O et al. 2003 Fibroblast growth factor 23 in oncogenic osteomalacia and X-linked hypophosphatemia. New England Journal of Medicine 348 1656-1663. (doi:10.1056/NEJMoa020881)

Kang HB, Kim JS, Kwon HJ, Nam KH, Youn HS, Sok DE \& Lee Y 2005 Basic fibroblast growth factor activates ERK and induces c-fos in human embryonic stem cell line MizhES1. Stem Cells and Development 14 395-401. (doi:10.1089/scd.2005.14.395)

Katoh M 2006 Cross-talk of WNT and FGF signaling pathways at GSK3beta to regulate beta-catenin and SNAIL signaling cascades. Cancer Biology and Therapy 5 1059-1064.

Kim E, Arnould T, Sellin LK, Benzing T, Fan MJ, Gruning W, Sokol SY, Drummond I \& Walz G 1999 The polycystic kidney disease 1 gene product modulates Wnt signaling. Journal of Biological Chemistry 274 4947-4953. (doi:10.1074/jbc.274.8.4947)
Kim YS, Kang YK, Kim JB, Han SA, Kim KI \& Paik SR 2000 ß-Catenin expression and mutational analysis in renal cell carcinomas. Pathology International 50 725-730. (doi:10.1046/j.1440-1827.2000.01111.x)

Kuro-o M 2006 Klotho as a regulator of fibroblast growth factor signaling and phosphate/calcium metabolism. Current Opinion in Nephrology and Hypertension 15 437-441. (doi:10.1097/01.mnh. 0000232885.81142.83)

Larsson T, Marsell R, Schipani E, Ohlsson C, Ljunggren O, Tenenhouse HS, Juppner H \& Jonsson KB 2004 Transgenic mice expressing fibroblast growth factor 23 under the control of the alpha1(I) collagen promoter exhibit growth retardation, osteomalacia, and disturbed phosphate homeostasis. Endocrinology 145 3087-3094. (doi:10.1210/en.2003-1768)

Liu S, Guo R, Simpson LG, Xiao ZS, Burnham CE \& Quarles LD 2003 Regulation of fibroblastic growth factor 23 expression but not degradation by PHEX. Journal of Biological Chemistry 278 37419-37426. (doi:10.1074/ jbc.M304544200)

Liu S, Tang W, Zhou J, Stubbs JR, Luo Q, Pi M \& Quarles LD $2006 a$ Fibroblast growth factor 23 is a counter-regulatory phosphaturic hormone for vitamin D. Journal of the American Society of Nephrology 17 1305-1315. (doi:10.1681/ASN.2005111185)

Liu S, Zhou J, Tang W, Jiang X, Rowe DW \& Quarles LD $2006 b$ Pathogenic role of Fgf23 in Hyp mice. American Journal of Physiology. Endocrinology and Metabolism 291 E38-E49. (doi:10.1152/ajpendo.00008.2006)

Liu S, Tang W, Fang J, Ren J, Li H, Xiao Z \& Quarles LD 2009 Novel regulators of Fgf23 expression and mineralization in Hyp bone. Molecular Endocrinology 23 1505-1518. (doi:10.1210/me.2009-0085)

Livak KJ \& Schmittgen TD 2001 Analysis of relative gene expression data using real-time quantitative PCR and the $2(-$ Delta Delta $C(T))$ method. Methods 25 402-408. (doi:10.1006/meth.2001.1262)

Lyles KW, Harrelson JM \& Drezner MK 1982 The efficacy of vitamin $D_{2}$ and oral phosphorus therapy in X-linked hypophosphatemic rickets and osteomalacia. Journal of Clinical Endocrinology and Metabolism 54 307-315. (doi:10.1210/jcem-54-2-307)

Marsell R, Krajisnik T, Goransson H, Ohlsson C, Ljunggren O, Larsson TE \& Jonsson KB 2008 Gene expression analysis of kidneys from transgenic mice expressing fibroblast growth factor-23. Nephrology, Dialysis, Transplantation 23 827-833. (doi:10.1093/ndt/gfm672)

Medici D, Razzaque MS, Deluca S, Rector TL, Hou B, Kang K, Goetz R, Mohammadi M, Kuro OM, Olsen BR et al. 2008 FGF-23-Klotho signaling stimulates proliferation and prevents vitamin D-induced apoptosis. Journal of Cell Biology 182 459-465. (doi:10.1083/jcb.200803024)

Raucci A, Bellosta P, Grassi R, Basilico C \& Mansukhani A 2008 Osteoblast proliferation or differentiation is regulated by relative strengths of opposing signaling pathways. Journal of Cellular Physiology 215 442-451. (doi:10. 1002/jcp.21323)

Shimada T, Mizutani S, Muto T, Yoneya T, Hino R, Takeda S, Takeuchi Y, Fujita T, Fukumoto S \& Yamashita T 2001 Cloning and characterization of FGF23 as a causative factor of tumor-induced osteomalacia. PNAS $\mathbf{9 8}$ 6500-6505. (doi:10.1073/pnas.101545198)

Shimada T, Hasegawa H, Yamazaki Y, Muto T, Hino R, Takeuchi Y, Fujita T, Nakahara K, Fukumoto S \& Yamashita T 2004a FGF-23 is a potent regulator of vitamin D metabolism and phosphate homeostasis. Journal of Bone and Mineral Research 19 429-435. (doi:10.1359/JBMR.0301264)

Shimada T, Kakitani M, Yamazaki Y, Hasegawa H, Takeuchi Y, Fujita T, Fukumoto S, Tomizuka K \& Yamashita T $2004 b$ Targeted ablation of Fgf23 demonstrates an essential physiological role of FGF23 in phosphate and vitamin D metabolism. Journal of Clinical Investigation 113 561-568. (doi:10. 1172/JCI19081)

Shimada T, Urakawa I, Yamazaki Y, Hasegawa H, Hino R, Yoneya T, Takeuchi Y, Fujita T, Fukumoto S \& Yamashita T 2004c FGF-23 transgenic mice demonstrate hypophosphatemic rickets with reduced expression of sodium phosphate cotransporter type IIa. Biochemical and Biophysical Research Communications 314 409-414. (doi:10.1016/j.bbrc.2003.12.102)

Sitara D, Razzaque MS, Hesse M, Yoganathan S, Taguchi T, Erben RG, Juppner H \& Lanske B 2004 Homozygous ablation of fibroblast growth factor-23 results in hyperphosphatemia and impaired skeletogenesis, and reverses hypophosphatemia in Phex-deficient mice. Matrix Biology 23 421-432. (doi:10.1016/j.matbio.2004.09.007) 
Stark K, Vainio S, Vassileva G \& McMahon AP 1994 Epithelial transformation of metanephric mesenchyme in the developing kidney regulated by Wnt-4. Nature 372 679-683. (doi:10.1038/372679a0)

ten Berge D, Brugmann SA, Helms JA \& Nusse R 2008 Wnt and FGF signals interact to coordinate growth with cell fate specification during limb development. Development 135 3247-3257. (doi:10.1242/dev.023176)

Tenenhouse HS \& Econs MJ 2001 Mendelian Hypophosphatemias.

In The Metabolic and molecular bases of Inherited Disease, Chapter 197: 1-9. Ed D Valle. New York: The McGraw-Hill Companies.

Urakawa I, Yamazaki Y, Shimada T, Iijima K, Hasegawa H, Okawa K, Fujita T, Fukumoto S \& Yamashita T 2006 Klotho converts canonical FGF receptor into a specific receptor for FGF23. Nature 444 770-774. (doi:10.1038/ nature05315)
Yamazaki Y, Okazaki R, Shibata M, Hasegawa Y, Satoh K, Tajima T, Takeuchi Y, Fujita T, Nakahara K, Yamashita T et al. 2002

Increased circulatory level of biologically active full-length FGF-23 in patients with hypophosphatemic rickets/osteomalacia. Journal of Clinical Endocrinology and Metabolism 87 4957-4960. (doi:10.1210/jc. 2002-021105)

Received in final form 20 July 2010

Accepted 23 July 2010

Made available online as an Accepted Preprint 30 July 2010 